\title{
Frequent Diagnostic Errors in Cardiac PET/CT Due to Misregistration of CT Attenuation and Emission PET Images: A Definitive Analysis of Causes, Consequences, and Corrections
}

\author{
K. Lance Gould ${ }^{1}$, Tinsu Pan $^{2}$, Catalin Loghin ${ }^{3}$, Nils P. Johnson ${ }^{4}$, Ashrith Guha ${ }^{5}$, and Stefano Sdringola ${ }^{6}$ \\ ${ }^{I}$ The Weatherhead PET Center for Preventing and Reversing Atherosclerosis, University of Texas Medical School and Memorial \\ Hermann Hospital, Houston, Texas; ${ }^{2}$ Imaging Physics Department, M.D. Anderson Cancer Center, University of Texas, Houston, Texas; \\ ${ }^{3}$ The Weatherhead PET Center and Division of Cardiology, The Department of Medicine, University of Texas Medical School at Houston, \\ Houston, Texas; ${ }^{4}$ Cardiology Division, Department of Medicine, Northwestern Medical School, Chicago, Illinois; ${ }^{5}$ Department of \\ Medicine, University of Texas Medical School at Houston, Houston, Texas; and ${ }^{6}$ The Weatherhead PET Center and Division of \\ Cardiology, Department of Medicine, University of Texas Medical School at Houston, Houston, Texas
}

Cardiac PET combined with CT is rapidly expanding despite artifactual defects and false-positive results due to misregistration of PET and CT attenuation correction data-the frequency, cause, and correction of which remain undetermined. Methods: Two hundred fifty-nine consecutive patients underwent diagnostic rest-dipyridamole myocardial perfusion $\mathrm{PET} / \mathrm{CT}$ using ${ }^{82} \mathrm{Rb}$, a 16-slice PET/CT scanner, helical CT attenuation correction with breathing and also at end-expiratory breath-hold, and averaged cine CT data during breathing. Misregistration on superimposed PET/CT fusion images was objectively measured in millimeters and correlated with associated quantitative size and severity of PET defects. Misregistration artifacts were defined as PET defects with corresponding misregistration on helical CT-PET fusion images that resolved after correct coregistration using a repeat CT scan, cine CT averaged attenuation during normal breathing, or shifted cine CT data that coregistered with PET data. Results: Misregistration of standard helical CT PET images caused artifactual PET defects in 103 of $259(40 \%)$ patients that were moderate to severe in $59(23 \%)(P=0.0000)$ and quantitatively normalized on cine or shifted cine CT PET $(P=0.0000)$. Quantitative misregistration was a powerful predictor of artifact size and severity $(P=0.0000)$, particularly for transaxial misregistration $>6 \mathrm{~mm}$ occurring in anterior or lateral areas in $76 \%$, in inferior areas in $16 \%$, and at the apex in $8 \%$ of 103 artifactual defects. Conclusion: Misregistration of helical CT attenuation and PET emission images causes artifactual defects with falsepositive results in $40 \%$ of patients that normalize on cine CT PET using averaged CT attenuation data during normal breathing comparable to normal breathing during PET emission scanning and shifting cine CT images to coregister visually with PET.

Received Jan. 14, 2007; revision accepted Apr. 17, 2007

For correspondence or reprints contact: K. Lance Gould, MD, The Weatherhead PET Center, University of Texas Medical School, 6431 Fannin St., Room 4.256MSB, Houston, TX 77030.

E-mail: K.Lance.Gould@uth.tmc.edu

COPYRIGHT @ 2007 by the Society of Nuclear Medicine, Inc.
Key Words: PET; image registration; heart; attenuation; artifact J Nucl Med 2007; 48:1112-1121

DOI: 10.2967/jnumed.107.039792

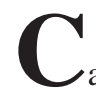
ardiac PET is an expanding noninvasive technology for identifying flow-limiting stenosis of coronary arteries, for assessing their severity as the basis of invasive procedures, for risk stratification, or for following progression or regression of coronary artery disease (CAD) after intense risk factor treatment that also predicts coronary events ( $1-$ 15). Mild reductions in relative coronary flow reserve by PET indicate early nonobstructive or diffuse CAD as the basis for treatment before significant stenosis develops (9). These applications of PET perfusion imaging require accurate, reliable differentiation of small regional differences in relative myocardial radionuclide uptake $(9,10)$.

Although thoracic and cardiac PET/CT has proven feasible, misregistration of attenuation and emission images causes artifactual abnormalities on cardiac PET images that are false-positive defects (16-24) or may artifactually worsen the severity of real perfusion defects. Misregistration of CT attenuation correction and PET emission data with associated artifactual PET defects is due to momentary helical CT "snapshots" at some point in the respiratory cycle of changing attenuating thoracic-diaphragmatic structures during breathing. The brief CT snapshots commonly do not match the actual average attenuation of the constantly changing thoracic-diaphragmatic structures over longer emission scans during normal breathing. This potentially major clinical limitation has not been definitively defined or resolved for current clinical protocols with commercially available cardiac PET/CT scanners and software. Thus, for 
PET/CT, the brief CT transmission data for attenuation correction are fundamentally different from earlier PET scanners using a rotating rod source for transmission data acquired slowly during normal breathing comparable to emission data acquisition.

Consequently, we examined the frequency, causes, consequences, and corrections of misregistration artifacts in current cardiac PET/CT protocols and software not previously reported, despite adverse effects on diagnostic accuracy.

\section{MATERIALS AND METHODS}

\section{Study Patients}

Two hundred fifty-nine consecutive patients undergoing diagnostic myocardial perfusion rest-dipyridamole PET/CT for potential CAD or follow-up imaging were analyzed at The Weatherhead PET Center for Preventing and Reversing Atherosclerosis of the University of Texas Medical School at Houston. All subjects signed an informed consent approved by the Committee for the Protection of Human Subjects of the University of Texas Health Science Center.

\section{PET}

Patients were instructed to fast for $4 \mathrm{~h}$ and abstain from caffeine, theophylline, and cigarettes for $24 \mathrm{~h}$ before the study. PET/CT was performed using a Discovery ST 16-slice PET/CT multislice bismuth germanate tomograph (GE Healthcare) in 2-dimensional mode with extended septa at a reconstructed inplane resolution of 5.9-mm full width at half maximum (FWHM). Patients were positioned in the scanner using laser guides aligned to the base of the throat and confirmed by a CT scout scan. External body markers were used to check correct position throughout data acquisition. CT transmission scans were obtained as detailed below. Emission images were then obtained over $6 \mathrm{~min}$ after intravenous injection of $1,295-1,850 \mathrm{MBq}(35-50 \mathrm{mCi})$ of generatorproduced ${ }^{82} \mathrm{Rb}$ and contained $24-60$ million total counts, of which 12-30 million were true coincidence counts.

Immediately after completing the resting ${ }^{82} \mathrm{Rb}$ scan, dipyridamole $(0.142 \mathrm{mg} / \mathrm{kg} / \mathrm{min})$ was infused for $4 \mathrm{~min}$. At 4 min after completion of the dipyridamole infusion, the same dose of ${ }^{82} \mathrm{Rb}$ was given intravenously. Emission image acquisition was started at $70 \mathrm{~s}$ after the beginning of ${ }^{82} \mathrm{Rb}$ infusion or $80 \mathrm{~s}$ after the beginning of infusion for patients with heart failure or heart rates below 55 beats per minute. After completion of stress emission imaging, a second helical CT transmission scan was obtained. For angina induced by dipyridamole, aminophylline $(125 \mathrm{mg})$ was given intravenously.

The first 145 consecutive patients were imaged using helical CT attenuation correction of the PET data to determine the extent and causes of misregistration associated with artifactual PET defects as falsely positive studies. Helical CT transmission scans were obtained at $120 \mathrm{kV}$ and $100 \mathrm{~mA}$ over $29 \mathrm{~s}$ during normal breathing in a subset of patients or over $4 \mathrm{~s}$ at end-expiratory breath-hold in another subset of patients. For the next 114 consecutive patients, in addition to the short end-expiratory helical CT, cine CT during normal breathing was added to the helical CT protocol. These subsets of patients served to compare quantitatively the extent of misregistration and associated artifactual defects between PET images reconstructed with helical CT attenuation correction data at end expiration and images reconstructed with cine CT averaged attenuation correction data during breathing in the same patient.

\section{Imaging Protocols, Data Acquisition, and Attenuation Correction}

After positioning in the gantry, patients had a CT scout scan using $120 \mathrm{kV}$ and $10 \mathrm{~mA}$ in anterior-posterior format to bracket the heart. A cine CT scan for average CT attenuation correction was acquired during normal breathing at rest; this was followed by a helical CT scan at end expiration. Perfusion imaging of ${ }^{82} \mathrm{Rb}$ was then obtained at rest and after dipyridamole stress; this was followed by another cine CT scan during normal breathing for stress average attenuation correction and another helical CT scan. The total time for a complete rest-stress study averaged $40 \pm 6$ $\min ($ mean $\pm 1.0 \mathrm{SD})$.

Cine-mode data acquisition is one of the 3 optional data acquisitions on the Discovery PET/CT scanner that include axial, helical, and cine modes. The helical CT acquisition used $120 \mathrm{kVp}$, $\mathrm{x}$-ray collimation of 16 by $1.25 \mathrm{~mm}$, gantry rotation of $0.5 \mathrm{~s}$, and helical pitch of 1.75:1. The radiation exposure was $5.7 \mathrm{mGy}$ for the helical CT scan.

The cine CT scan used $120 \mathrm{kVp}$, x-ray collimation of 8 by 2.5 $\mathrm{mm}$, gantry rotation cycle of $0.5 \mathrm{~s}$, cine duration of $10 \mathrm{~s}$, and 14$\mathrm{cm}$ coverage. Each reconstruction used $360^{\circ}$ of CT data over $0.5 \mathrm{~s}$. The cine data acquisition lasted for $78 \mathrm{~s}$, of which $70 \mathrm{~s}$ was for 7 cine CT acquisitions of $10 \mathrm{~s}$ each and $8 \mathrm{~s}$ was for 6 table translations of $2 \mathrm{~cm}$ each between 2 cine CT acquisitions. Tube current for cine CT was $10 \mathrm{~mA}$ for patients weighing $<100 \mathrm{~kg}, 15 \mathrm{~mA}$ for $100-130 \mathrm{~kg}$, and $20 \mathrm{~mA}$ for $>130 \mathrm{~kg}$. The radiation dose and effective dose equivalent for cine CT were $10 \mathrm{mGy}$ and $2.36 \mathrm{mSv}$, respectively, for tube current of $10 \mathrm{~mA}$. For each cine CT scan, there are 1,232 CT images reconstructed 2 min after the cine CT acquisition and 22 images per slice location during normal respiration before averaging into an average CT image over several breathing cycles. The processing time for an averaged cine CT attenuation scan was $<1$ min.

Interpolation of the average CT attenuation data was performed to make the average CT images align at the locations of the PET slices for attenuation correction of the PET data and for evaluating registration between the average CT image and the PET images corrected with the average CT attenuation data. In view of the inherent blurring introduced by the average $\mathrm{CT}$ but not by the helical CT data, we applied 4- and 10-mm FWHM gaussian filtering on the average CT and helical CT data, respectively, before CT attenuation correction of the PET data.

Software was also developed to shift the averaged cine CT data in horizontal and vertical directions in the transaxial plane and in the superior-inferior or $z$-axis direction of different planes to align the CT heart borders visually with the PET data to achieve good coregistration. The average cine CT slices shifted to match the PET slices are saved for repeat reconstruction of the PET images using the shifted average attenuation correction data. The cine CT PET has been previously described for cancer imaging (21), here adapted to cardiac imaging with the shift software.

\section{Analysis of PET Images}

Images were reconstructed using filtered backprojection with a Butterworth filter having a cutoff of 0.55 , roll-off of 10 , and pixel size of $3.27 \times 3.27 \mathrm{~mm}$. Fusion images were made by superimposing attenuation-corrected emission and CT transmission scans in horizontal, coronal, and sagittal views. Misregistration 
of CT and PET images was quantified in millimeters for comparison with quantitative size and severity of regional perfusion defects on PET images corresponding to the regions of misregistration on the PET/CT fusion display.

After attenuation correction, reconstructed PET emission images in DICOM (Digital Imaging and Communications in Medicine) format were exported to a Sun Ultra 60 workstation of a mPower-HZL PET scanner (Positron Corp.) for reorientation into long- and short-axis tomographic and topographic 3-dimensional (3D) displays using previously described automated quantitative software $(1-10)$ for comparison with our earlier report on misregistration artifacts in PET using a rotating rod transmission source for attenuation correction (20). Quantitative data from ${ }^{82} \mathrm{Rb}$ and ${ }^{18} \mathrm{~F}$ phantoms imaged on the GE Healthcare scanner are quantitatively and accurately exported to the Positron scanner and software for automated quantitative analysis and display of PET images.

A 3D restructuring algorithm generates true short- and longaxis views from reconstructed PET transaxial cardiac images, perpendicular to and parallel to the long axis of the left ventricle. From the tomographic data, circumferential profiles are used to reconstruct $3 \mathrm{D}$ topographic views of the left ventricle showing relative regional activity distribution divided into lateral, inferior, septal, and anterior quadrant views of the 3D topographic display corresponding to the coronary arteries as previously described $(4-10,20)$.

Mean activity in each quadrant is normalized to the maximum $2 \%$ of pixels in the whole heart dataset. Regions of each quadrant are identified having values outside $2.0 \mathrm{SD}$ and $2.5 \mathrm{SD}(97.5 \%$ confidence intervals [CI]) outside normal values of 50 healthy volunteers with no risk factors by complete medical history (no hypercholesterolemia, hypertension, diabetes, smoking, obesity, drug or alcohol abuse, other systemic diseases, or family history of CAD). Percentages of circumferential profile units outside 2.0 SD and 2.5 SD were calculated automatically for each quadrant and the whole heart.

Severity of a perfusion defect is quantified as the average relative uptake in a defined quadrant or the lowest quadrant average relative activity - that is, the average relative activity for the quadrant having the lowest average activity of anterior, septal, lateral, and inferior quadrants for each subject, expressed as percentage of the highest $2 \%$ of activity in the image dataset. Size of perfusion defects is quantified as percentage of the whole cardiac image outside 2.0 SD or $2.5 \mathrm{SD}$ or $97.5 \% \mathrm{CI}$ of healthy control subjects in each quadrant and whole heart. Combined size and severity of perfusion defects is defined as percentage of the whole cardiac image with relative activity of $<60 \%$ of maximum activity (100\%), which is $3.0 \mathrm{SD}$ below mean maximum activity of healthy control subjects.

\section{Misregistration Artifacts}

On fusion images of helical CT PET and separately of cine CT PET, electronic calipers were used to measure the distance in millimeters between the cardiac border on the PET image and the cardiac border on the CT image in the horizontal plane of maximal misregistration on transaxial and coronal views and in the vertical or $z$-axis of the coronal view. In addition, on the coronal view, the distance was measured from the zero reference position of helical and cine CT scans to the dome of the right diaphragm to determine systematically the effects of diaphragmatic position on misregistration artifacts.
On PET perfusion images, artifactual abnormalities due to misregistration were defined as criteria (i) plus (ii), (iii), or (iv) as follows:

(i) The defect on the PET image was associated with a corresponding area of misregistration on the superimposed CT transmission and PET emission fusion scans.

(ii) An abnormality on the resting CT PET scan associated with misregistration disappeared, and the image normalized with correct coregistration of CT and PET emission data on the stress PET scan.

(iii) The abnormalities on the helical CT PET scans at rest or stress disappeared, and the image normalized with correct coregistration of CT and PET emission data on the cine CT PET scan or when the misregistration was corrected by shifting the cine CT scan to coregister visually with the PET scan.

(iv) The abnormality on helical CT PET disappeared on an additional separate rest-stress study repeated on the next day using an mPower-HZL standard PET scanner with a rotating rod transmission source and shift software to ensure correct registration of emission and transmission data as previously documented (20).

Therefore, to be counted as having an abnormality due to PET/ CT misregistration, every patient with abnormal helical CT PET at rest or stress had to have a stress perfusion study with no significant regional defect after correct PET/CT coregistration. Any patient with a defect that persisted on stress PET/CT images or after correct coregistration that was outside 2 SD of healthy subjects was classified as having a "real" defect, not an artifact, even if the defect improved after correct registration. Therefore, our observed prevalence of abnormalities due to PET/CT misregistration is a conservative underestimate.

For PET scans meeting the above criteria for misregistration artifacts, the severity, size, and combined size-severity of perfusion abnormalities in the same quadrant as the CT PET misregistration were objectively quantified by automated software. In addition to automated measures of defect size and severity, the perfusion abnormalities due to CT PET misregistration were visually graded according to the relative color scale bar by 2 independent readers, who were unaware of clinical information, as none (white or red), mild (yellow), moderate (green), or severe (blue) in the same quadrant for which misregistration was measured.

\section{Statistical Methods}

Statistical analyses were performed using SPSS version 11.5 (SPSS Inc.) for multivariate linear regression analysis, for multivariate logistic regression analysis, and for significance of differences in continuous variables among groups determined by a paired or unpaired 2-tailed $t$ test with data reported as mean \pm 1 SD. A Pearson $\chi^{2}$ analysis was performed for discrete variables. A 2-tailed $P$ value $<0.05$ was considered statistically significant.

\section{RESULTS}

Figure 1A illustrates an abnormal PET scan with a mildto-moderate anterior and lateral defect (top row) due to misregistration of helical CT transmission and PET data. This defect disappears and the image normalizes after repeat reconstruction using cine $\mathrm{CT}$ attenuation correction 


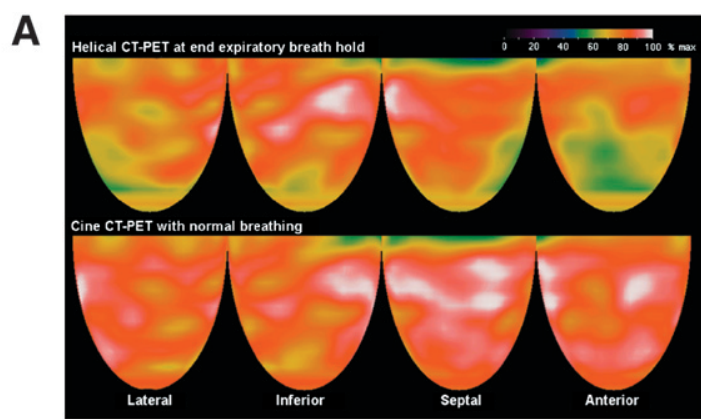

B

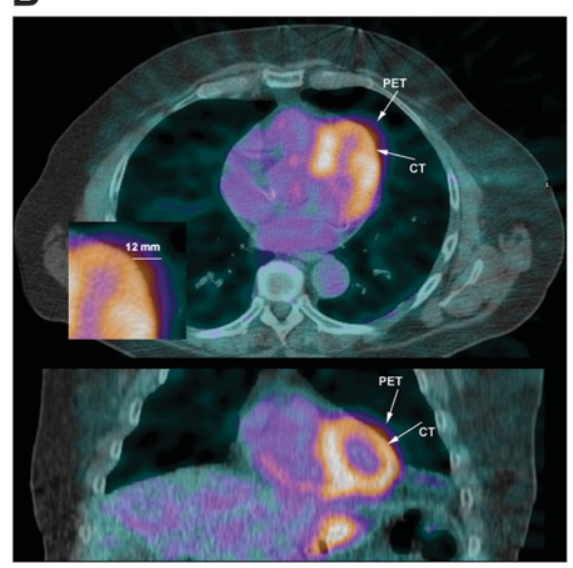

FIGURE 1. (A) Topographic 3D displays of helical CT PET with a mild-to-moderate anterior and lateral defect (top row) that is not present on cine CT PET (bottom row). White indicates the highest myocardial uptake of ${ }^{82} \mathrm{Rb}$, reflecting the highest myocardial perfusion, with red being the next highest and progressively lower perfusion indicated by color gradations from red to yellow, green, and blue. (B) For same patient as in A, misregistration on helical CT-PET fusion images in transaxial (top) and coronal (bottom) views. Arrows indicate heart borders on helical CT and PET emission images as unmatched, with region of misregistration corresponding to area of artifactual defect. Magnified inset illustrates quantification of misregistration in transaxial view-here, $12 \mathrm{~mm}$ - using an electronic caliper on the screen. (C) For same patient, cine CT-PET fusion images show good coregistration associated with no defect and a normal scan. (bottom row). Figure 1B shows the corresponding helical CT-PET fusion images with severe misregistration in transaxial and coronal views but good coregistration on the cine CT-PET fusion images (Fig. 1C). The magnified inset illustrates quantification of misregistration in the transaxial view using an image caliper, $12 \mathrm{~mm}$ in this example.

The quantitative extent of misregistration of the helical CT attenuation correction data and the PET emission data is analyzed on a per-patient basis by $\chi^{2}$ analysis in Table 1. With misregistration of $>6 \mathrm{~mm}$ on the transaxial fusion images of helical CT and PET emission images, the PET perfusion scan showed artifactual defects that were significant diagnostic errors in 103 of 259 (40\%) patients $(P=$ $0.0000)$. Transaxial misregistration of $>6 \mathrm{~mm}$ frequently caused artifactual defects with the proportion of moderate-

TABLE 1

All Patients

\begin{tabular}{lrrrrr}
\hline & \multicolumn{5}{c}{$\begin{array}{c}\text { Artifactual PET defects due to } \\
\text { Transaxial } \\
\text { misregistration }\end{array}$} \\
\cline { 2 - 6 } misregistration $(\mathrm{mm})$ & None & Mild & Moderate & Severe & Total \\
\hline$\leq 6$ & 148 & 4 & 2 & 0 & 154 \\
$>6-10$ & 7 & 22 & 19 & 2 & 50 \\
$>10$ & 1 & 18 & 29 & 7 & 55 \\
Total & 156 & 44 & 50 & 9 & 259 \\
& & & & & \\
\hline$\chi^{2} P=0.0000$. & & & & & \\
\hline
\end{tabular}

to-severe artifactual defects progressively increasing for misregistration of $6-10 \mathrm{~mm}$ and for $>10 \mathrm{~mm}$.

Of 259 patients, 137 were classified as having real perfusion abnormalities outside $2 \mathrm{SD}$ of healthy subjects that persisted after correction of PET/CT misregistration or on the stress PET and, therefore, were not counted as artifactual abnormalities. The remaining 122 patients with no persisting defects after stress or after correct PET/CT coregistration were analyzed quantitatively to compare those with and without misregistration artifacts (as defined in Materials and Methods). For quantitative data in Table 2, these strict criteria removed patients with real defects outside 2 SD of healthy control subjects on the final averaged cine or averaged cine shifted stress CT PET images, thereby eliminating the bias of real defects on quantification of the artifactual defects due to misregistration. Visually mild, moderate, and severe artifactual defects (as defined in Materials and Methods) showed statistically significant progressive worsening in severity, in combined size-severity, in size as percentage $<2 \mathrm{SD}$ and in size as percentage $<2.5$ $\mathrm{SD}$ of healthy subjects with severity of PET/CT misregistration in millimeters.

In 59 patients (23\% of the 259), the artifactual defects due to misregistration on helical CT PET images were moderate to severe and significantly outside normal limits for quantitative severity, size, and combined size-severity. This prevalence of artifactual abnormalities due to PET/CT misregistration is a conservative underestimate of true prevalence because a real defect in any patient outside 2 SD of 
TABLE 2

Size and Severity of Misregistration Artifacts

\begin{tabular}{|c|c|c|c|c|c|c|}
\hline PET/CT $(n=122)$ & Severity & Size-severity & $\% \leq 2.0 \mathrm{SD}$ & $\% \leq 2.5 \mathrm{SD}$ & $\begin{array}{c}\text { Transaxial } \\
\text { misregistration (mm) }\end{array}$ & $n$ \\
\hline NA & $79.6 \pm 3.1$ & $0.1 \pm 0.2$ & $2.5 \pm 3.1$ & $0.8 \pm 1.1$ & $2.4 \pm 2.4$ & 19 \\
\hline Artifacts & $73.9 \pm 6.4$ & $6.2 \pm 14.6$ & $8.6 \pm 4.9$ & $6.1 \pm 4.9$ & $11.7 \pm 5.0$ & 103 \\
\hline$P$ for $\Delta$ & 0.0000 & 0.0000 & 0.0000 & 0.0000 & 0.0000 & \\
\hline Mild artifact & $75.9 \pm 3.9$ & $1.8 \pm 3.6$ & $6.8 \pm 3.9$ & $3.9 \pm 3.5$ & $10.2 \pm 3.8$ & 44 \\
\hline$P$ for $\Delta$ vs. NA & 0.0003 & 0.0024 & 0.0000 & 0.0000 & 0.0000 & \\
\hline Moderate artifact & $74.2 \pm 5.3$ & $3.8 \pm 5.3$ & $9.3 \pm 5.0$ & $6.8 \pm 4.7$ & $12.1 \pm 5.0$ & 50 \\
\hline$P$ for $\Delta$ vs. NA & 0.0000 & 0.0000 & 0.0000 & 0.0000 & 0.0000 & \\
\hline Severe artifact & $62.9 \pm 10.3$ & $41.07 \pm 31.1$ & $13.9 \pm 3.8$ & $12.3 \pm 4.8$ & $16.5 \pm 7.3$ & 9 \\
\hline$P$ for $\Delta$ vs. NA & 0.0011 & 0.0043 & 0.0000 & 0.0001 & 0.0003 & \\
\hline No or mild artifact & $77.0 \pm 4.1$ & $1.3 \pm 3.1$ & $5.5 \pm 4.2$ & $2.9 \pm 3.3$ & $7.9 \pm 4.9$ & 63 \\
\hline Moderate or severe artifact & $72.4 \pm 7.4$ & $9.5 \pm 18.4$ & $10.0 \pm 5.1$ & $7.7 \pm 5.1$ & $12.8 \pm 5.6$ & 59 \\
\hline$P$ for $\Delta$ vs. no or mild & 0.0001 & 0.0013 & 0.0000 & 0.0000 & 0.0000 & \\
\hline
\end{tabular}

healthy subjects that persisted on stress images was not counted as an artifactual defect even if it improved after correct PET/CT registration.

Logistic multivariate regression analysis was done with the 3 misregistration measurements in millimeters (transaxial $x$-axis, coronal horizontal $x$-axis, and coronal vertical $z$-axis) as independent variables, with the dependent variable being separately either any artifact or only moderate-to-severe artifacts. Transaxial misregistration ( $x$-axis) predicted misregistration artifactual defects so completely (coefficient of regression analysis, $\mathrm{B}=0.94, P=0.0000$ ) that the other measurements added no more predictive power.

Similarly, multivariate regression analysis was done with the 3 misregistration measurements as independent variables and the dependent variable being separately the severity (mean quadrant activity), size (percentage outside 2.5 SD), or combined size-severity (percentage of the left ventricle outside $60 \%$ of maximum activity) of the abnormalities due to PET/CT misregistration. The coronal horizontal ( $x$-axis) misregistration measurement was such a strong predictor of the 3 quantitative measures of defect severity $(\mathrm{B}=2.23$ for combined size-severity, $\mathrm{B}=0.96$ for severity, $\mathrm{B}=0.59$ for percentage outside $2.5 \mathrm{SD}$; for all, $P=0.0000$ ) that other misregistration measurements added no predictive power. These results indicate that the 3 misregistration measurements are closely related such that the $x$-axis misregistration in either the transaxial or coronal view is a complete predictor of artifactual defects.

For patients with both helical and cine CT PET (Table 3), the artifactual defects due to misregistration of helical CT attenuation and PET emission data were markedly reduced by using the averaged cine $\mathrm{CT}$ data acquired during normal breathing that is comparable to the PET emission acquisition during normal breathing. Compared with all patients, this quantitative improvement was even greater for the subset of patients with artifactual defects due to PET/CT misregistration, excluding those with real defects as defined above. Transaxial PET/CT misregistration in millimeters was significantly less with cine CT PET compared with helical CT PET.

Diaphragm position was significantly lower on averaged cine CT images than on helical CT at end expiration, indicating that the diaphragm at end expiration was higher than the average diaphragmatic position during breathing. This higher diaphragmatic position at end expiration caused more misregistration artifacts than slower helical CT during breathing and more than averaged cine CT during breathing as detailed below.

Most misregistration defects were anterior or lateral (76\%) associated with corresponding anterior or lateral misregistration on the CT-PET fusion images. The remainder had inferior $(8 \%)$ or apical $(16 \%)$ abnormalities due to inferior or apical misregistration of helical CT PET or different diaphragmatic position during the helical CT compared with the diaphragm position on the cine CT average attenuation scan that resolved the artifactual defect. Both the artifactual defects and their associated misregistration on helical CT-PET fusion images resolved on cine CT PET in most cases.

However, of the 114 patients with both helical CT and cine CT PET, 22 (19\%) had persisting artifactual defects with persisting PET/CT misregistration on the cine CT-PET fusion images. For these patients, the cine CT data were shifted to achieve proper visual coregistration with the PET images that were then reconstructed again with the shifted cine CT attenuation data. With this final coregistration, the artifactual defect disappeared and the images normalized.

Figure 2A illustrates severe anterior, apical, lateral, and basal inferior defects on the helical CT PET (top row) that 
TABLE 3

Patients with Both Helical and Cine CT PET

\begin{tabular}{|c|c|c|c|c|c|c|c|c|}
\hline PET/CT scan & $\begin{array}{l}\text { Severity (\% of } \\
\text { maximum) }\end{array}$ & $\begin{array}{c}\text { Size-severity } \\
(\%<60 \% \\
\text { maximum) }\end{array}$ & $\begin{array}{l}\% \text { of LV } \\
<2.0 \mathrm{SD}\end{array}$ & $\begin{array}{c}\% \text { LV } \\
<2.5 \mathrm{SD}\end{array}$ & $\begin{array}{c}\text { Transaxial } \\
\text { misregistration } \\
(\mathrm{mm})\end{array}$ & $\begin{array}{c}\text { Mean } \Delta \text { cine } \\
\text { vs. helical }(\mathrm{mm})\end{array}$ & $\begin{array}{l}\text { Diaphragm } \\
\mathrm{mm} \text { to CT } 0\end{array}$ & $\begin{array}{c}\text { Mean } \Delta \mathrm{mm} \\
\text { diaphragm }\end{array}$ \\
\hline All helical CT PET & $74.2 \pm 7.3$ & $8.5 \pm 17.5$ & $8.2 \pm 5.8$ & $6.1 \pm 5.6$ & $8.34 \pm 6.51$ & 4.4 & $5.2 \pm 15.8$ & 3.3 \\
\hline All cine CT PET & $77.6 \pm 6.2$ & $4.5 \pm 11.6$ & $5.1 \pm 5.1$ & $3.5 \pm 4.7$ & $3.93 \pm 3.50$ & & $2.0 \pm 15.4$ & \\
\hline$n=114, P$ for $\Delta$ & 0.0000 & 0.0024 & 0.0000 & 0.0000 & 0.0000 & & 0.0002 & \\
\hline $\begin{array}{l}\text { Helical CT PET } \\
\text { with artifacts }\end{array}$ & $73.7 \pm 7.3$ & $7.5 \pm 17.6$ & $8.7 \pm 5.4$ & $6.3 \pm 5.3$ & $11.87 \pm 5.89$ & 7.2 & $5.3 \pm 16.9$ & 2.6 \\
\hline $\begin{array}{l}\text { Cine CT PET } \\
\text { same patients }\end{array}$ & $79.2 \pm 5.0$ & $1.2 \pm 3.3$ & $3.9 \pm 3.8$ & $2.2 \pm 3.0$ & $4.69 \pm 3.67$ & & $2.7 \pm 16.2$ & \\
\hline$n=67, P$ for $\Delta$ & 0.0000 & 0.0038 & 0.0000 & 0.0000 & 0.0000 & & 0.0334 & \\
\hline
\end{tabular}

$\mathrm{LV}=$ left ventricle.

Quantitative measurements of defects are for quadrant containing misregistration artifactual defect or for worst quadrant in absence of misregistration artifact.

remained but were less severe on cine CT PET (middle row). The anterior, apical, and lateral defects resolved only on the shifted cine PET/CT (bottom row) that achieved proper coregistration. However, the basal inferior defect persisted with correct registration, indicating right coronary stenosis, confirmed by coronary arteriography with no stenosis of the left coronary artery. Figures 2B-2D show the corresponding misregistration on the helical CT PET and cine CT-PET fusion images but no misregistration on the shifted cine CT-PET fusion images.
A

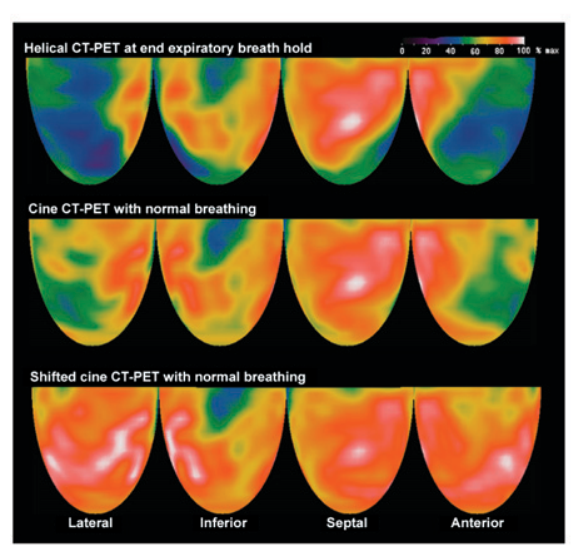

C

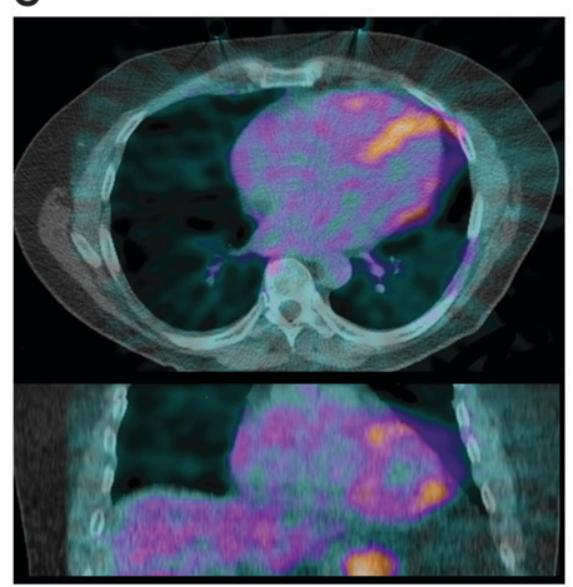

B

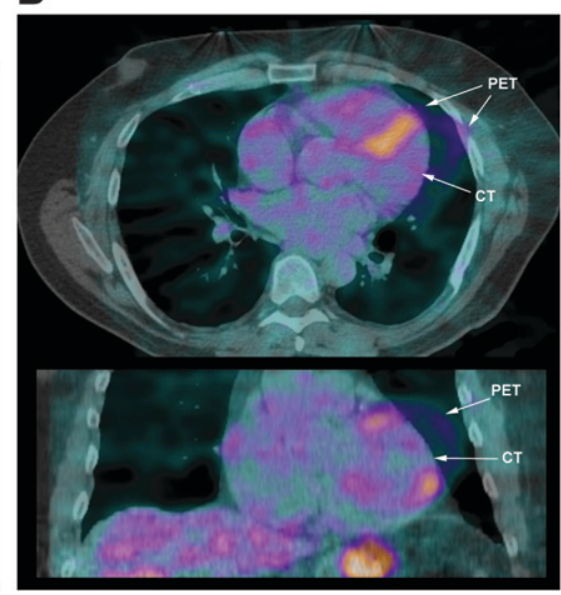

D

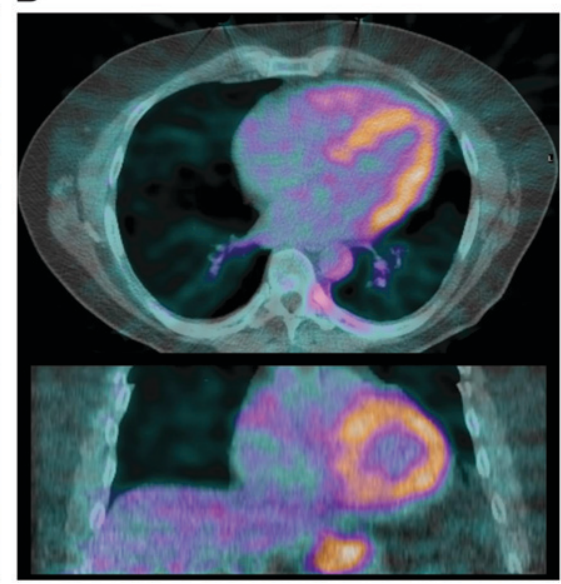

FIGURE 2. (A) Topographic 3D displays of helical CT PET with severe anterior, apical, lateral, and basal inferior defects (top row) that are also present but less severe on cine CT PET (middle row). Anterior, apical, and lateral defects normalize on shifted cine CT PET (bottom row). (B) For same patient as in A, helical CT-PET fusion images in transaxial (top) and coronal (bottom) views show marked misregistration. Arrows indicate heart borders on helical CT and PET emission images as unmatched, with region of misregistration corresponding to area of artifactual defect. (C) For same patient, cine CT-PET fusion images also show misregistration. (D) For same patient, shifted cine CT-PET fusion images with no misregistration associated with disappearance of artifactual anterior, apical, and lateral artifactual defects. 
TABLE 4

Patients with Cine CT PET Having Worse Misregistration Artifactual Defects than with Helical CT PET Corrected by Shifting Cine CT Data

\begin{tabular}{|c|c|c|c|c|}
\hline $\begin{array}{l}\text { Cine CT PET worse than } \\
\text { helical CT (8 patients) }\end{array}$ & $\begin{array}{l}\text { Severity (minimum } \\
\text { quadrant average) }\end{array}$ & $\begin{array}{l}\text { Size-severity ( } \% \\
<60 \% \text { maximum) }\end{array}$ & Size $(\% \leq 2.5 \mathrm{SD})$ & $\begin{array}{c}\text { Transaxial } \\
\text { misregistration }(\mathrm{mm})\end{array}$ \\
\hline Helical CT PET & $74.79 \pm 3.60$ & $1.72 \pm 2.58$ & $5.26 \pm 4.49$ & $8.39 \pm 3.00$ \\
\hline Cine CT PET & $71.28 \pm 5.45$ & $9.64 \pm 10.27$ & $10.08 \pm 5.57$ & $12.44 \pm 5.51$ \\
\hline$n=8, P$ for $\Delta$ & 0.0216 & 0.0284 & 0.0018 & 0.0751 \\
\hline Cine CT PET & $70.29 \pm 5.07$ & $11.01 \pm 10.27$ & $10.88 \pm 5.49$ & $12.24 \pm 5.93$ \\
\hline Shifted cine CT PET & $75.98 \pm 6.10$ & $4.05 \pm 6.60$ & $4.98 \pm 5.77$ & $2.71 \pm 3.39$ \\
\hline$n=7^{\star}, P$ for $\Delta$ & 0.0043 & 0.0639 & 0.0047 & 0.0008 \\
\hline
\end{tabular}

${ }^{*}$ Quantitative shifted cine CT data were lost for 1 patient due to corruption of the header but with good saved images.

Of the 22 patients in whom cine CT failed to correct misregistration, 8 of the 114 patients with both helical CT and cine CT PET (7\%) showed more severe misregistration on cine CT PET than on helical CT PET, with a corresponding more severe artifactual defect on cine CT PET images. These 8 artifactual defects were corrected by reconstructing the PET images after shifting the cine CT average attenuation images to coregister visually with the PET images with corresponding quantitative improvement and normalization of the images (Table 4).

Artifactual defects may also be caused by the diaphragm being in a different position during helical CT and PET despite good coregistration of the heart borders on CT-PET fusion images (Fig. 3), obtained before cine CT was available. In this patient with known CAD, the rest-dipyridamole
PET of 1999 with a rotating rod attenuation source showed moderately severe regional stress perfusion defects that improved at yearly follow-up after intense medical treatment (Fig. 3A).

In 2006, on the same regimen with no symptoms or change in clinical status or medications, follow-up helical CT PET showed apparent marked worsening with new, moderately severe anterior and distal inferior stress defects not present previously. Helical CT-PET fusion images showed proper coregistration of heart borders but demonstrated a markedly different heart-to-diaphragm relation (Fig. 3B). The heart is in the same location on both rest and stress CT and PET scans according to the CT reference position marked by the red cross-hairs. However, because the patient was breathing, the diaphragm on the stress

FIGURE 3. (A) Stress perfusion images at yearly intervals using a Positron PET scanner with rotating rod transmission source show progressive improvement on a strict lifestyle and medical regimen. (B) For same patient as in A, rest and stress helical CT-PET fusion images at follow-up in 2006 were acquired during normal breathing before cine CT was available. Diaphragm-heart mismatch (arrows) on stress fusion image caused attenuation overcorrection inferiorly and associated relative anterior defect on stress PET despite borders of heart being properly coregistered on both rest and stress perfusion images (see text). (C) For same patient, follow-up stress perfusion helical CT PET images in 2006. Diaphragm-heart mismatch on stress fusion image shown in B caused artifactual anterior, lateral, and inferoapical defects (top row) that disappeared on repeated stress scan using Positron PET scanner with a rotating rod attenuation transmission source (bottom row) and correct coregistration.

A

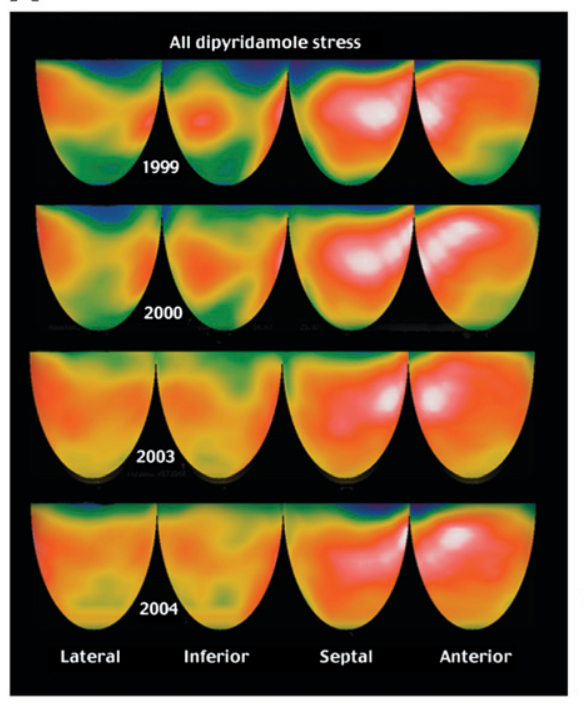

B

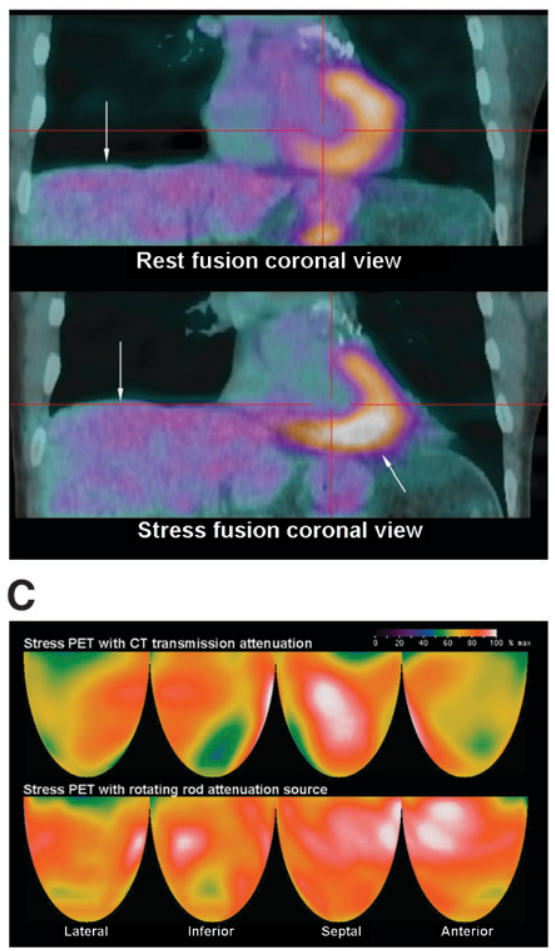


helical CT is much higher relative to the heart on the stress fusion image, whereas the heart on the stress PET image remained unchanged from rest.

This diaphragm-heart mismatch on the stress fusion image caused attenuation overcorrection inferiorly and an associated relative anterior defect on the stress PET. In addition, the stomach air bubble is higher on the expiratory helical CT than the inferior heart border on the emission scan, thereby creating a small strip of inferior attenuation undercorrection despite the rest of the inferior half of the heart being overcorrected.

To resolve the question of severe worsening before cine CT was available for this patient, the PET study was repeated on the next day with the same rest-dipyridamole protocol, same medications, and same dose of ${ }^{82} \mathrm{Rb}$ but on the Positron PET scanner with a rotating rod attenuation source. This repeat rest-stress study showed no misregistration and no significant stress-induced perfusion defects and was similar to the earlier PET scans, displayed for comparison with the helical CT PET of the prior day in Figure 3C. The absence of significant perfusion defects after correct coregistration indicates artifactual defects on the earlier PET/CT.

We also examined the frequency of artifactual defects due to misregistration in patients with slow 29-s helical CT scans during breathing compared with different patients with fast 4-s helical CT scans during end-expiratory breathholding. Slow helical PET/CT during breathing had significantly fewer artifactual defects due to misregistration than fast helical PET/CT at end-expiratory breath-hold (Table 5). In addition, the artifactual defects with slow helical CT PET during breathing were of a different type associated with layering of the CT data and multiple diaphragm and heart border fragments, which caused jagged notched layers of CT attenuation correction data. Correspondingly, the artifactual defects consisted of jagged, irregular linear notches or bites out of the resting CT and PET perfusion images as illustrated in Figure 4.

\section{DISCUSSION}

This study demonstrates artifactual defects on cardiac helical CT PET in $40 \%$ of patients that are false-positive

TABLE 5

Patients with Slow Helical CT over 29 Seconds During Breathing Compared with Patients with Fast 4-Second Helical CT at End-Expiratory Breath-Holding

\begin{tabular}{ccccc}
\hline Comparison & No artifacts & Artifacts & Total & $\%$ artifacts \\
\hline $\begin{array}{c}\text { Slow helical CT } \\
\text { with breathing }\end{array}$ & 106 & 39 & 145 & 27 \\
$\begin{array}{c}\text { Fast helical CT at } \\
\text { end expiration }\end{array}$ & 50 & 64 & 114 & 56 \\
Total & 156 & 103 & 259 & 40 \\
\hline$\chi^{2} P=0.001$. & & & & \\
\hline
\end{tabular}

results. These artifactual defects are of 3 types: (a) PET/CT misregistration, (b) diaphragm-heart PET/CT mismatching, and (c) layering of helical CT data seen with slow helical CT scans during breathing. These misregistration abnormalities are due to short helical CT snapshots in the respiratory cycle of attenuating structures that fail to match the average attenuation of constantly changing thoracicdiaphragmatic structures during breathing over longer emission scans. They can be recognized and quantified on superimposed CT PET fusion images and corrected by cine CT PET protocols or by software for shifting the averaged cine CT data to coregister visually with the PET images.

Although slow helical CT scans during breathing have somewhat less-frequent artifactual defects than fast helical CT scans at end expiration, the layered attenuation data of the long helical CT during breathing distorts the PET images in a way that cannot be corrected by shifting the CT data to coregister with the PET data. Similarly, shifting the short helical CT data at end expiration to coregister with the PET data is also unsatisfactory because CT attenuation is greater during expiration and, therefore, causes attenuation overcorrection compared with the average attenuating structures during breathing as measured by cine CT during breathing.

Compared with our earlier study of PET attenuation misregistration using a rotating rod attenuation source during breathing, artifactual defects in helical CT PET are more frequent, more severe, and more complex. Preventing or correcting these artifactual defects in PET/CT requires cine CT and software for shifting the CT data to visually coregister with the PET data when needed.

Some early thoracic or cardiac studies on small numbers of patients reported satisfactory CT attenuation correction of PET emission data $(16,19,22,23)$, whereas others reported problems of misregistration $(17,18,24)$. A recent study on 28 patients reported substantial artifactual perfusion defects due to misregistration of PET/CT data in $21 \%$ of subjects (24). Visual realignment of PET/CT data to achieve coregistration eliminated the artifactual perfusion defects. However, our results indicate that a helical CT scan at one moment during the respiratory cycle may not fit the average attenuating structures during breathing of emission imaging even after visually matching the lateral heart borders.

Moreover, variable attenuation correction associated with respiratory motion causes marked heterogeneity in measurements of myocardial radionuclide uptake (22). Therefore, measurements of absolute myocardial perfusion in $\mathrm{mL} / \mathrm{min} / \mathrm{g}$ by PET/CT are appropriate only if the primary radionuclide update data are correctly obtained using cine or shifted cine CT attenuation correction that matches the average attenuation structures of emission data during breathing. We believe that the relatively poor specificity reported for PET/CT (13) is due to misregistration artifacts as no protocol for correcting misregistration of PET/CT data was described in that study.

Artifacts in Cardiac PET/CT • Gould et al. 
A

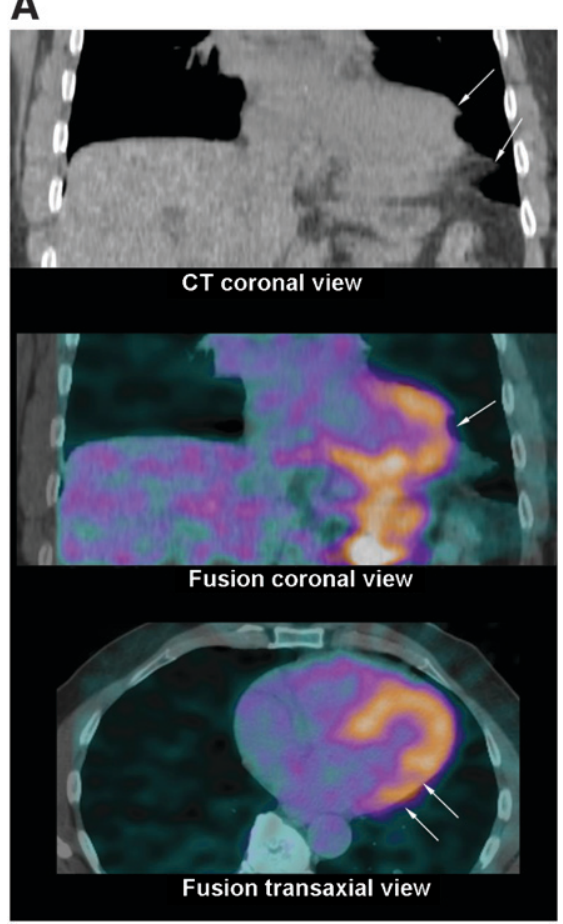

B

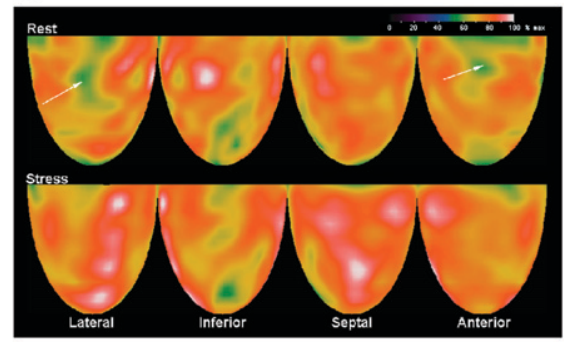

FIGURE 4. (A) Resting CT transmission image acquired over $29 \mathrm{~s}$ with normal breathing shows "motion layering" of CT scan (top row). Helical CT-PET fusion image demonstrates a corresponding notching of attenuation (arrows) and notched undercorrection of PET data. (B) For same patient as in A, corresponding rest-stress $3 \mathrm{D}$ topographic display has linear anterior and lateral artifacts (arrows) on rest emission scan caused by motion layering on resting CT scan, not present on stress scan. Mild inferior defect is not associated with any misregistration but is due to diffuse atherosclerosis of posterior descending coronary artery.
One criticism of our study may be the lack of coronary arteriography to document the absence of stenosis. However, invasive coronary arteriography is not justified for a defect in a region of PET/CT misregistration that disappears after repeat reconstruction using correctly coregistrered CT attenuation data, particularly in view of well-documented inaccuracies of visually interpreted coronary arteriograms.

Our software implementation is applicable to all Discovery PET/CT scanners without any additional hardware. GE Healthcare provides an expensive hardware upgrade of their PET/CT console to the Dimension console that achieves some of the functions reported here. However, the Dimension console averages combined helical and cine CT data for attenuation correction, provides no average cine CT images for shifting to register with PET data, and is available only on the latest Discovery STE PET/CT. Other manufacturers do not have the interface for cine CT. Alternatively, a non-CT pure PET scanner with a 3-rod rotating attenuation source would acquire attenuation data very rapidly without the misregistration and relatively high radiation dose of PET/CT, thereby providing the basis for an inexpensive fast PET scanner for cardiac imaging.

\section{CONCLUSION}

Misregistration of helical CT attenuation and emission PET images is common in cardiac PET/CT, with associated artifactual PET defects causing falsely positive results that are identifiable on PET/CT fusion images. Artifactual defects due to PET/CT misregistration are eliminated by imaging protocols using cine CT data acquired during normal breathing and software for reconstructing PET images with averaged cine $\mathrm{CT}$ data or sifting averaged cine $\mathrm{CT}$ data to achieve visual coregistration. Properly coregistered PET/ CT data provide definitive perfusion images demonstrated in this study and, as reported earlier, suitable for assessing severity of CAD, for following its regression or progression, and for identifying mild early nonobstructive coronary atherosclerosis as the basis for intense, life-long pharmacologic and lifestyle management $(1-10)$.

\section{ACKNOWLEDGMENT}

No author has a conflict of interest or financial benefit related to this article.

\section{REFERENCES}

1. Demer LL, Gould KL, Goldstein RA, Kirkeeide RL. Diagnosis of coronary artery disease by positron emission tomography: comparison to quantitative coronary arteriography in 193 patients. Circulation. 1989;79:825-835.

2. Sdringola S, Patel D, Gould KL. High prevalence of myocardial perfusion abnormalities by positron emission tomography in asymptomatic people having a parent or sibling with coronary artery disease. Circulation. 2001;103:496-501.

3. Nakagawa Y, Nakagawa K, Sdringola S, Mullani N, Gould KL. A precise three dimensional atlas of myocardial perfusion correlation with coronary arteriographic anatomy. J Nucl Cardiol. 2001;8:580-590.

4. Gould KL, Martucci JP, Goldberg DI, et al. Short-term cholesterol lowering decreases size and severity of perfusion abnormalities by positron emission tomography after dipyridamole in patients with coronary artery disease. Circulation. 1994;89:1530-1538.

5. Gould KL, Ornish D, Scherwitz L, et al. Changes in myocardial perfusion abnormalities by positron emission tomography after long-term, intense risk factor modification. JAMA. 1995;274:894-901.

6. Sdringola S, Nakagawa K, Nakagawa Y, et al. Combined intense lifestyle and pharmacologic lipid treatment further reduce coronary events and myocardial perfusion abnormalities compared with usual-care cholesterol-lowering drugs in coronary artery disease. J Am Coll Cardiol. 2003;41:263-272. 
7. Sdringola S, Loghin C, Boccalandro F, Gould KL. Mechanisms of progression and regression of coronary disease by positron emission tomography related to treatment intensity and risk of clinical events at long-term follow-up. $\mathrm{J} \mathrm{Nucl}$ Med. 2006;47:59-67.

8. Gould KL. Assessing progression or regression of CAD: the role of perfusion imaging. J Nucl Cardiol. 2005;12:625-638.

9. Gould KL, Nakagawa Y, Nakagawa N, et al. Frequency and clinical implications of fluid dynamically significant diffuse coronary artery disease manifest as graded, longitudinal, base-to-apex, myocardial perfusion abnormalities by noninvasive positron emission tomography. Circulation. 2000;101:1931-1939.

10. Johnson NP, Gould KL. Clinical evaluation of a new concept: resting myocardial perfusion heterogeneity quantified by markovian analysis of PET identifies coronary microvascular dysfunction and early atherosclerosis in 1,034 subjects. J Nucl Med. 2005;46:1427-1437.

11. Bateman TM, Heller GV, McGhie AI, et al. Diagnostic accuracy of rest/stress ECG-gated Rb-82 myocardial perfusion PET: comparison with ECG-gated Tc99m sestamibi SPECT. J Nucl Cardiol. 2006;13:24-33.

12. Dorbala S, Hassan A, Heinonen T, Schelbert HR, Di Carli MF. Coronary vasodilator reserve and Framingham risk scores in subjects at risk for coronary artery disease. J Nucl Cardiol. 2006;13:761-767.

13. Sampson UK, Dorbala S, Limaye A, Kwong R, Di Carli MF. Diagnostic accuracy of rubidium-82 myocardial perfusion imaging with hybrid positron emission tomography/computed tomography in the detection of coronary artery disease. J Am Coll Cardiol. 2007;49:1052-1058.

14. Chow BJ, Wong JW, Yoshinaga K, et al. Prognostic significance of dipyridamoleinduced ST depression in patients with normal ${ }^{82} \mathrm{Rb}$ PET myocardial perfusion imaging. J Nucl Med. 2005;46:1095-1101.
15. Yoshinaga $\mathrm{K}$, Chow BJ, Williams $\mathrm{K}$, et al. What is the prognostic value of myocardial perfusion imaging using rubidium- 82 positron emission tomography? J Am Coll Cardiol. 2006;48:1029-1039.

16. Yu JN, Fahey FH, Harkness BA, Gage HD, Eades CG, Keyes JW Jr. Evaluation of emission-transmission registration in thoracic PET. J Nucl Med. 1994;35:1777-1780.

17. Matsunari I, Boning G, Ziegler SI, et al. Effects of misalignment between transmission and emission scans on attenuation-corrected cardiac SPECT. J Nucl Med. 1998;39:411-416.

18. McCord ME, Bacharach SL, Bonow RO, Dilsizian V, Cuocolo A, Freedman N. Misalignment between PET transmission and emission scans: its effect on myocardial imaging. J Nucl Med. 1992;33:1209-1214.

19. Koepfli P, Hany TF, Wyss CA, et al. CT attenuation correction for myocardial perfusion quantification using a PET/CT hybrid scanner. J Nucl Med. 2004;45: 537-542.

20. Loghin C, Sdringola S, Gould KL. Common artifacts in PET myocardial perfusion images due to attenuation-emission misregistration: clinical significance, causes and solutions in 1177 patients. J Nucl Med. 2004;45:1029-1039.

21. Pan T, Mawlawi O, Luo D, et al. Attenuation correction of PET cardiac data with low-dose average CT in PET/CT. Med Phys. 2006;33:3931-3938.

22. Le Meunier L, Maass-Moreno R, Carrasquillo J, Dieckmann W, Bacharach SL. PET/CT imaging: effect of respiratory motion on apparent myocardial uptake. J Nucl Cardiol. 2006;13:821-830.

23. Gilman MD, Fischman AJ, Krishnasetty V, Halpern EF, Aquino SL. Optimal CT breathing protocol for combined thoracic PET/CT. AJR. 2006;187:1357-1360.

24. Martinez-Moller A, Souvatzoglou M, Navab N, Schwaiger M, Nekolla SG. Artifacts from misaligned CT in cardiac perfusion PET/CT studies: frequency, effects, and potential solutions. J Nucl Med. 2007;48:188-193. 